\title{
Video in science
}

\author{
Protocol videos: the implications for research and society
}

\author{
Matias Pasquali
}

T he Chinese folktale, Three Blind Men and an Elephant, is more than 2,000 years old. Three blind men approached an elephant and tried to describe it. The first man felt the elephant's ear and decided that it was like a fan. The second man touched the elephant's knee and decided that it was like a tree. The third man held the elephant's trunk and concluded that it was like a snake. They could not agree on what an elephant is really like (Kuo \& Kuo, 1976). If each of the three men had felt the whole of the elephant, or at least experienced each of the three parts themselves, they would have gained a better idea of what animal they were trying to describe.

Scientists, like the blind men, often have too narrow a focus when thinking about their field of research or particular topics. To obtain a larger, more complete picture-to understand what the 'elephant' is-they need as much information as possible. One way of achieving this is to take advantage of new technologies, such as video, and use it to communicate scientific methods, protocols and results. Here, I describe the possible advantages of using video to communicate scientific protocols instead of verbal descriptions, and highlight some further benefits of such an approach, in particular in educational and outreach programmes.

V isual information is fundamental to communication. As humans, we have relied on our visual perception of the world to convey ideas, feelings and images ever since our ancestors started painting animals on cave walls thousands of years ago. Drawings, paintings, photography, film, television, analogue and digital video, computer-generated images and the Internet have all further increased and enhanced the use of images to communicate information-not just in general, but specifically with regard to scientific ideas, results and communication.

Human vision is the most highly developed of our senses; therefore, images are a particularly efficient method of communicating information. The part of the brain devoted to processing and analysing input from the eyes is larger than the parts devoted to processing the input from any other sense. Indeed, "[T]he perceptual power of the image may also be seen in its ability to dominate the written or spoken word when they appear together. In fact both written and oral forms of language must be cognitively processed first, whereas images are perceptually processed along the same alternative pathways as direct experience" (Barry, 1997). Given this highly developed image-processing system-which outclasses the most sophisticated image-processing software-it makes sense to use images to convey large amounts of information in a relatively short space of time-a task made easier by modern means of creating and manipulating image and video data.

Scientists began using moving images to record and describe their work almost as soon as the technology became available. One of the pioneers of 'scientific' films was the French physiologist Étienne-Jules Marey, who built the 'photographic rifle' in 1882-a forerunner of the movie camera-for his studies of animal and human locomotion. He used his gun-like camera to film the flight of seagulls, achieving a frame-rate of 12 photographs per second. The French scientist was nicknamed 'Posillipo's madman' by the Neapolitans because they saw him taking careful aim at the birds with his odd rifle without firing a shot (Tosi, 1986).
During the early twentieth century, the technological development of 'moving images' was driven more by scientific interests than commercial or artistic ones. For anthropologists in particular, who had previously had to record foreign cultures as narratives-and whatever had been hard to put into words was lost - the recording of moving images was a huge leap forward in their methods of data collection (Mead, 1963). Other scientists also began to explore the use of moving images: the zoologists Roberto Omegna and Francis Martin Duncan; the surgeon Francesco Pasinetti, who filmed 35 operations; the British naturalists Percy Smith and Mary Field, who created the series Secrets of Nature from 1922 to 1933; and France's Jean Painlevé, who produced more than 200 films, mainly about natural history. In addition, the French scientist, Jean Comandon, pioneered microcinematography in 1908, by coupling the ultramicroscope-the precursor of today's dark-field microscopes-to a camera in order to film cells, bacteria, protozoans and fungi.

\section{Scientists began using moving images to record and describe their work almost as soon as the technology became available}

Since these early scientific movies, the sophistication of their use in scientific work has increased markedly. The use of static and moving images and the ability to manipulate and share them through the World Wide Web has revolutionized scientific procedures, enhanced our ability to discover new things and offered new opportunities for education. 
I $\mathrm{n}$ our increasingly visually oriented age, scientific research and education rely on the use of visual tools to present technical information-today's students live in an environment saturated with images. Video is a valuable teaching tool because it can be used to show students things that would be otherwise hard to organize 'live'. For example, veterinary or medical surgery, biological experiments and their results, laboratory procedures and anthropological documentaries are all now shown through video feeds or hosted on the Internet. Similarly, the video streaming of seminars or interviews with scientists, together with additional information on their current research-for example, the Nature Online Video Streaming Archive-have improved the quality of scientific education. Videoconferences enable virtual meetings; video delivers real-time lectures to students-even when they are on placement in ambulatory clinics to hospitals in rural areas, or to students at other medical schools (Oz, 2005). In the same way, diagnostic infrastructures coupled with video streaming allow experts to identify and analyse pathogens remotely (Stack et al, 2006).

In addition to teaching and learning, an increasing number of scientists use video to present their results at scientific meetings, during lectures or in their publications as online supplementary material. Some research groups also present their work as videos that can be downloaded from their own websites; for example, the laboratories of Angus Lamond (http:// www.lamondlab.com/f7home.htm) and Julie Theriot (http://cmgm.stanford.edu/theriot/ movies.htm). Pascal Wallisch, a graduate student at the University of Chicago (IL, USA) recently attracted the attention of the press by attaching video iPods to his poster during a meeting of the American Society for Neuroscience. The iPods showed an animated presentation of his work (Anon, 2006).

Now that both the Internet and the hardware for exchanging videos have achieved sufficient bandwidth and reliability (Häder, 2001), many laboratories in various fields are using videos to document their research data. Scientific journals increasingly include videos as supplementary data to provide information that cannot be communicated by text alone. Recent examples include a film of the vibration and rotation of the nucleus of a hydrogen molecule as a quantum mechanical wave packet (Ergler et al, 2006), a study of the behaviour of aggressive mosquitoes (Dierick \& Greenspan, 2006) and the use of time-lapse video microscopy to monitor the morphological change of cellular organelles (Julian, 2007).

T he 'materials and methods' section is an essential part of any research article, but it has always been difficult to reproduce some experiments by relying solely on the published text. To improve the efficacy of the communication and reproduction of experiments, new formats have been developed, for example, the new online-only full methodology descriptions for articles in Nature (Anon, 2007). Yet video could be an even more efficacious remedy to the problem of 'showing' scientists how to reproduce experiments (Pasquali, 2006). Indeed there are various initiatives to make protocols freely available online, including publishing videos of the procedures involved. Websites such as Nature Protocols, Cold Spring Harbor Protocols, OpenWetWare and DNAtube offer both the software and the server space for hosting movies of protocols (Pearson, 2006). Despite the potential of this format, only a few websites currently integrate text and movies to provide a more detailed description of scientific methods (Ledford, 2006). A promising development is the launch of the Journal of Visual Experiments (JoVE; Kritikou, 2007), an online journal that publishes biological experiments as videos that include step-by-step instructions and short discussions concerning possible technical problems.

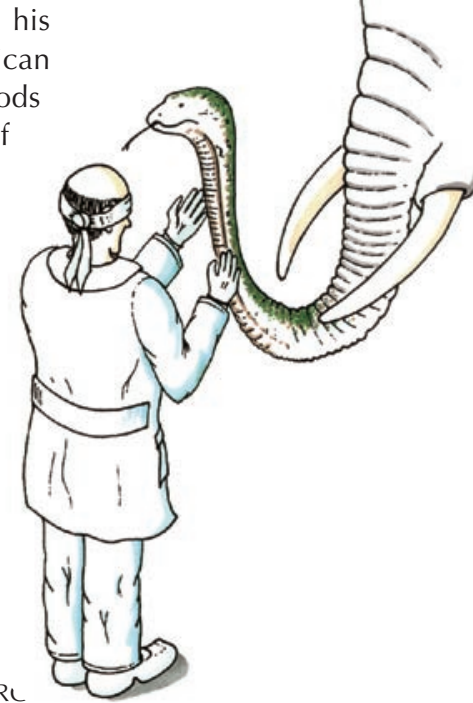

Clearly, there are various practical advantages to publishing scientific methods as videos. Because video includes information such as colour, position, duration, shape and motion, it is the optimal format for transmitting the manifold details of new protocols or technical procedures. Communicating these details through prose would require considerable page-space-which is usually not available in scientific journals - and a lack of page-space often results in an article summarizing a procedure and omitting crucial details that are needed to replicate the experiment. Implicitly, this also diminishes transparent communication. The use of video can therefore improve scientific communication by fulfilling a crucial basic requirement of scientific experiments: their reproducibility. For example, microarray experiments, based on tissues or whole organisms, require the communication of an enormous amount of detail to effectively replicate the experiment. The adoption of video descriptions might therefore further improve the Minimum Information About a Microarray Experiment (MIAME) standard (Brazma et al, 2001).

Describing methodologies by using video can improve the critical analysis of scientific procedures in two ways. First, when experimenters observe their own procedures on video, they can examine their research from an external point of view - this 'external observer' condition is essential for science (Alrøe \& Kristensen, 2002). Second, peer review of methodologies could be expanded in a way that has not been possible with written descriptions of experiments.

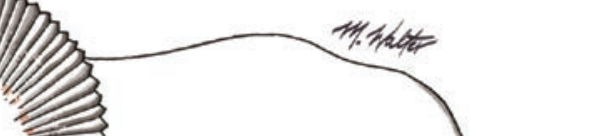


In fact, the representation of a methodology can only really be shown in enough detail by video and, as such, video enhances the amount of information presented and allows a more accurate judgement to be made on the correctness of a procedure.

\section{As video includes information such as colour, position, duration, shape and motion, it is the optimal format for transmitting the manifold details of a new protocol or for explaining complex technical procedures}

However, the use of video would require changes to be made to the 'classical' peer-review process. Reviewers would have to become more familiar with visual language and be able to recognize digital effects that might hint at image manipulation. They would also need to evaluate the clarity of the explanation of the method, as well as its completeness-for example by judging the integration of the image with subtitles or voiceover and by assessing the possibility of reproducing the experiment after watching the video.

The widely publicized stem-cell cloning fraud involving a Korean scientist, WooSuk Hwang, was first revealed by an anonymous Korean biologist who pointed out duplications in some of the photographs in Hwang's Science paper (Kennedy, 2006). Clearly it would not have been as easy for Hwang to perpetrate the fraud if the submitted article had included the videotaped creation of the 11 cell lines. Although dishonest scientists will always find ways to avoid the checks of the peer-review system, the live recording of scientific methods would make it more difficult to fabricate data or to obtain it inappropriately. Nevertheless, the use of video would not exclude fraud per se. It is still possible to manipulate or alter a video-taped proto$\mathrm{col}$, although it would require more sophisticated methods of cheating than simply altering the bands on a gel.

Another potential problem with video methodologies that scientists would have to be aware of are video artefacts that might skew the interpretation of results. To the untrained eye, such artefacts can present themselves as qualities or traits of the depicted objects, whereas in fact they are merely properties of the instrumentation.
Moreover, as with digital photographs, digital video would greatly increase the opportunity to alter or manipulate images-even if such manipulations were not intended to be dishonest. Although a video will show 'what actually happened', it "might allow scientists to express their vision or theory by means of the manner of recording and subsequent editing process [...] that is, at the root of every presentation of facts there is an implicit or explicit theory, a particular way of looking" (Pauwels, 2006). The particular field of research, cultural background, scientific tradition, circumstances of the experiment, skills or preferences of the researcher and the specific purpose of the video might all introduce subtle variations or subjective biases that could skew the interpretation of the results.

It is clear that the usefulness and reliability of video protocols will depend on the intentions and the integrity of the scientist who produces them. Without a doubt, video can add important information and valuable details to written descriptions and static images, and improve the ability of scientists to learn new methods, reproduce experiments or understand phenomena. But, as with any visual media, the credibility of a video relies on the honesty and integrity of the author, the reliability and robustness of the method of its recording, the trustworthiness of the publishers, and the controls and standards operating in the scientific community.

$\mathrm{T}$ he main challenge to the widespread adoption of a video format for presenting data and methodology is not the cost-prices for digital recording and editing systems have decreased considerably_or the extra time required; according to JOVE, a video can usually be produced in two days. The main deterrent against publishing videos is that highly competitive scientific activities entail a component of secrecy. Compared with written or verbal descriptions, video is far more revealing of the 'tricks of the trade' and makes it easier for others to exactly reproduce the author's experiments or copy a new procedure. Filming protocols might therefore affect the current trend for high levels of competition in scientific research. If video protocols further act to reverse the culture of secrecy that hinders the disclosure of valuable information, then their benefits might be greater than previously imagined.
However, there is also the question of security when filming protocols that deal with possible dual-use research, the results of which could be abused by criminals or terrorists. In such cases - which are rather rare-an increased level of censorship or secrecy might be desirable (van Aken, 2006). Yet many journals already have rules and committees to assess the level of security needed for such research and the same system could be implemented with regard to video protocols (Atlas \& Dando, 2006).

\section{... as with any visual media, the credibility of a video relies on the honesty and integrity of the author, the reliability and robustness of the method of its recording, the trustworthiness of the publishers, and the controls and standards operating in the scientific community}

Competitiveness and secrecy aside, the adoption of a video format for presenting new protocols and procedures would support the basic standards of communalism and organized scepticism that guide the practice of empirical research (Merton, 1973). Communalist science is a social activity built on previous efforts, and organized scepticism requires the social evaluation of claims through open debate, peer review and the duplication of work (Rabinow, 1996). Both of these norms require the efficient and precise documentation of past activities, and science thrives as a spontaneous activity wherever information is free and shared. The scientific endeavour should spread novel approaches in the most efficient and accessible way because they facilitate new applications and innovations.

M any scientists-more or less correctly-believe that the way science is portrayed in popular culture, in particular by television and cinema, has been detrimental to the public understanding of science (Van, 1995; Leslie, 2002; Tyson, 2002). The scientific facts are often factually wrong, scientists are portrayed as evil or at least socially inept, and scientific knowledge as intrinsically dangerous. According to the science and engineering indicators of the US National Science Foundation, the representation of 


\section{The use of video to record the work of laboratories might therefore be an effective way to portray an accurate view of science to society}

science by the media has generally corroded the ability of the public to think critically and has hindered scientific literacy. "The amount of information now available can be overwhelming and seems to be increasing exponentially. This has led to 'information pollution', which includes the presentation of fiction as a fact. Thus, being able to distinguish fact from fiction has become just as important as knowing what is true and what is not" (National Science Board, 2000).

In order to reverse this trend, scientists have made an effort to communicate more directly with the public, often through films and videos. Scientists often cooperate with film-makers to promote a more accurate depiction of science and work to revive a long tradition of making scientific documentaries. Video is an effective tool for communicating complex scientific ideasmoving images and colours easily attract the viewer's attention and are well suited to the task of explaining scientific concepts. One example of a grassroots movement to create videos recording scientific research is the annual 'Cinema of the Cell' festival, created by the French biologist Christian Sardet. Since 2002, the festival has taken place during the annual meeting of the European Life Scientist Organization (ELSO; Sandhausen, Germany) and is a forum for informative, entertaining 'bioclips' for teaching and communicating science to the general public (Breithaupt, 2002).

The use of video to record the work of laboratories might therefore be an effective way to portray an accurate view of science to society. By enabling the public to observe a cloning procedure or a microarray experiment, the concept becomes less obscure, and the mysterious and frightening becomes the mundane and ordinary. Studies of the effect of seeing an experiment on a person's perception of science have to be carried out, but exploring new methods of communication is essential to attract the public's attention to everyday scientific topics (Leshner, 2007).

In addition, the use of video can highlight aspects of science that are outside the boundaries of normal scientific reports, such as the daily realities of scientific research, its frustrations and successes. In a video by Hao Yuan Kueh (Monitoring actin disassembly with timelapse microscopy, http://www.myjove.org), the subtitle when the experiment succeeds reads "expression of happiness when experiment works", to reinforce images of the scientist smiling. This reveals a great deal about the human aspect of scientific endeavour.

t ournals such as JOVE, and freely available websites, are valuable tools for teaching science in schools and universities because they provide educators with access to the latest experiments to help stimulate curiosity and interest. But, in addition to watching scientific videos, students should learn to produce them. Filming an experiment, editing the video into a logical sequence, and adding audio commentary and/or subtitles can help to improve their understanding of the process of experimentation. Furthermore, it would stimulate students to realize that videos can be an important feature of research and would introduce a visual component that is still relatively neglected by teachers (Lowe, 2000).

\section{...the use of video can highlight aspects of science that are outside the boundaries of normal scientific reports, such as the daily realities of scientific research, its frustrations and successes}

In the short-term, the scientific community might not be too inclined to add videorecordings of methodologies to their repertoire because the change from the century-old practice of simply writing them down will require time and effort. The advantage of the more accurate and reproducible descriptions afforded by video might therefore be the best incentive for adopting this media, and better access to video protocols might also convince scientists to try this model for verifying and publishing their own findings. The success of initiatives such as JOVE, and the general acceptability of video methods in scientific journals will depend on the quality of the peer-review process for video submissions. To avoid 'information pollution', the videos published should be relevant to understanding the results of the research and offer high-quality information.

The other prerequisite to the success of using video to describe methods will be the willingness of scientists to adopt a different media-that is, not prose or graphics-and acquire a new 'language' with which to describe experiments (Pauwels, 2006). However, judging from the quality of the videos already available on the web, it seems that this is not a big problem-many laboratories and individuals are already presenting their work in this format. Modern technological advancements continually change and improve the tools available to scientists-both for communication and research-so there seems little reason not to embrace new video-sharing technologies. Scientists can prepare themselves for the revolution by grabbing a video camera and, in a few years, maybe there will be an Oscar for the best scientist 'director' of the year.

\section{ACKNOWLEDGEMENTS}

I thank Iris Reale for her suggestions and help with bibliographic material and the Branco Weiss Fellowship 'Society-in-Science' for support.

\section{REFERENCES}

Alrøe HF, Kristensen ES (2002) Towards systemic research methodology in agriculture. Rethinking the role of values in science. Agric Human Values 19: $3-23$

Anon (2006) Newsmakers: the first podster. Science 314: 1365

Anon (2007) Editorial: methods in full. Nature 445: 684

Atlas RM, Dando M (2006) The dual-use dilemma for the life sciences: perspectives, conundrums, and global solutions. Biosecur Bioterror 4: 276-286

Barry AM (1997) Visual Intelligence Perception, Image, and Manipulation in Visual Communication. Albany, NY, USA: State University of New York Press

Brazma A et al (2001) Minimum information about a microarray experiment (MIAME) — toward standards for microarray data. Nat Genet 29: 365-371

Breithaupt H (2002) Rhythm ' $n$ ' biology. EMBO Rep 3: 813-815

Dierick HA, Greenspan RJ (2006) Molecular analysis of flies selected for aggressive behaviour. Nat Genet 38: 1023-1031

Ergler Th, Rudenko A, Feuerstein B, Zrost K, Schröter CD, Moshammer R, Ullrich J (2006) Spatiotemporal imaging of ultrafast molecular motion: collapse and revival of the $\mathrm{D}_{2}{ }_{2}$ nuclear wave packet. Phys Rev Lett 97: 193001

Häder D-P (2001) Image Analysis: Methods and Applications. Boca Raton, FL, USA: CRC

Julian A (2007) Hiscox RNA viruses: hijacking the dynamic nucleolus. Nat Rev Microbio/ 5: 119-127

Kennedy D (2006) Editorial retraction. Science 311: 335

Kritikou E (2007) Watch and learn. Nat Rev Mol Cell Biol 8: 4

Kuo L, Kuo YH (1976) Chinese Folk Tales. Millbrae, CA, USA: Celestial Arts

Ledford H (2006) YouTube for test tubes. Nature news [doi:10.1038/news061120-12] 
Leshner AI (2007) Outreach training needed. Science 315: 161

Leslie M (2002) Hollywood howlers. Science 297: 19

Lowe R (2000) Visual literacy and learning in science. ERIC Digest Nov. www.ericdigests.org [ED463945]

Mead M (1963) Anthropology and the camera. In The Encyclopedia of Photography. The Complete Photographer: The Comprehensive Guide and Reference for all Photographers, WD Morgan (ed). New York, NY, USA: Greystone

Merton RK (1973) The Sociology of Science: Theoretical and Empirical Investigations. Chicago, IL, USA: University of Chicago Press National Science Board (2000) Science and Engineering Indicators-2000. Arlington, VA, USA: National Science Foundation

$\mathrm{Oz} \mathrm{HH}$ (2005) Synchronous distance interactive classroom conferencing. Teach Learn Med 17: 269-273
Pasquali M (2006) Videos have starring role to play in protocol sharing. Nature 443: 632

Pauwels L (2006) Visual Cultures of ScienceRethinking Representational Practices in Knowledge Building and Science Communication. Hanover, NH, USA: Dartmouth College Press

Pearson H (2006) Online methods share insider tricks. Nature 441: 678

Rabinow P (1996) Making PCR: A Story of Biotechnology. Chicago, IL, USA: University of Chicago Press

Stack J et al (2006) The national plant diagnostic network. Plant Dis 90: 128-136

Tosi V (1986) I/ Linguaggio delle Immagini in Movimento. Teoria e Tecnica del Cinema e della Televisione nella Ricerca Scientifica, Didattica e nella Divulgazione. Rome, Italy: Armando

Tyson N (2002) Hollywood nights: when it comes to astronomical accuracy in the movies, nobody's getting any Oscars. Natural History June: $26-31$
Van J (1995) Mad scientists. Chicago Tribune, 6 November, page C1

van Aken J (2006) When risk outweigh benefits. EMBO Rep 7: S10-S13

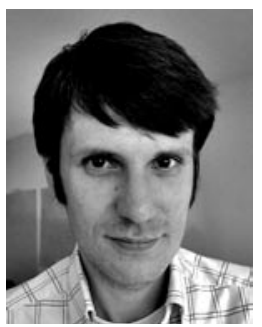

Matias Pasquali is Branco Weiss Fellow at the Department of Plant Pathology, University of Minnesota, St Paul, MN, USA E-mails: matias.pasquali@gmail.com, pasqu016@umn.edu

doi:10.1038/sj.embor.7401037 